\title{
Severity of Coronary Artery Disease by Coronary CT Angiography in Relation to Left Ventricular Diastolic Function
}

\author{
Wassam Eldin Hadad Elshafey ${ }^{1}$, Walaa Fareed Abdelaziz' ${ }^{1}$, Mohamed Seleem Mohamed², \\ Osama Mohamed Abdallah omar $^{2}$ \\ ${ }^{1}$ Cardiology Department, Menoufia University, Al Minufya, Egypt \\ ${ }^{2}$ National Heart Institute, Embaba, Cairo, Egypt \\ Email: Dr.osamaomarr@gmail.com
}

How to cite this paper: Elshafey, W.E.H., Abdelaziz, W.F., Mohamed, M.S. and Omar, O.M.A. (2020) Severity of Coronary Artery Disease by Coronary CT Angiography in Relation to Left Ventricular Diastolic Function. World Journal of Cardiovascular Diseases, 10, 235-245.

https://doi.org/10.4236/wjcd.2020.104022

Received: February 29, 2020

Accepted: April 26, 2020

Published: April 29, 2020

Copyright $\odot 2020$ by author(s) and Scientific Research Publishing Inc. This work is licensed under the Creative Commons Attribution International License (CC BY 4.0).

http://creativecommons.org/licenses/by/4.0/

\begin{abstract}
Objectives: The aim of this study is to evaluate the relationship between severity of CAD by coronary mutlislice computed tomography scan and the degree of diastolic function Methods: The study consisted of 80 patients who underwent both coronary CTA and TTE within 1 year with no identifiable intervening cardiovascular event in National Heart Institute and Menoufia University Hospitals. All patients were subjected to detailed medical history, physical examination, full electrocardiography study including tissue Doppler imaging on mitral annulus, MSCT coronary angiography and different laboratory investigation as CBC, creatinine, lipid profile. Results: From 80 patients who have done both echocardiography examination and MSCT coronary angiography, the CT scan results were 38 patients with non-obstructive coronary artery disease and 42 patients with obstructive $\mathrm{CAD}$, higher number of patients with diastolic dysfunction with higher values of E/E', medial e', lateral e', LAVI (left atrial volume index) and TRV (tricuspid regurge velocity) were found in the group with obstructive coronary artery disease. Conclusion: Our study showed that diastolic dysfunction is more prevalent in patients with obstructive coronary artery disease with higher grade of diastolic dysfunction.
\end{abstract}

\section{Keywords}

Coronary Artery Disease, Diastolic Dysfunction, MSCT Coronary Angiography

\section{Introduction}

Diastolic dysfunction (DD) is an important cause of heart failure (HF) with preserved ejection fraction ( $\mathrm{pEF}$ ) and a major public health issue. Epidemiological 
studies indicate that varying severities of DD are present in the community. DD is predictive of developing overt $\mathrm{HF}$ and all-cause mortality [1].

With advancing age and acquired cardiac conditions, such as hypertensive heart disease or coronary artery disease (CAD), LV relaxation may worsen and LA pressures increase, and despite preservation of LV systolic function, the clinical syndrome of diastolic heart failure (HF), otherwise known as HF with preserved ejection fraction (HFpEF), may develop [1].

Abnormal diastolic function of the left ventricle (LV) detected by transthoracic echocardiography (TTE), is associated with worse prognosis [2]. Diastolic function (DD) is the first cardiac function to be impaired in ischemic heart disease [3].

Early detection of LV diastolic dysfunction has important diagnostic and prognostic and therapeutic implications. Echocardiography is the most important feasible noninvasive method to evaluate LV DD, because of the inaccuracy of conventional pulsed wave mitral inflow Doppler analysis and having multiple limits and the inconclusive findings to diagnose DD. Tissue Doppler imaging is significantly sensitive and conclusive modality to get the velocity of the longitudinal motion of the mitral valve annulus and has the ability to early detect and confirm the diagnosis of left ventricular diastolic dysfunction [4].

Ischemic heart disease is one of the utmost causes of mortality and disability in the world, although coronary heart disease mortality rates have declined worldwide over the past decades, it remains the cause of for about one third or more of all-cause mortality in individuals above 40 years old [5].

MSCT angiography is a noninvasive scanning modality that permits the diagnosis of coronary arteries atherosclerosis. Since rapidly evolving technology results in better quality scanning and accurate for the diagnosis of coronary artery disease, despite the fact that MSCT is still less accurate than invasive coronary angiography due to lower resolution, significant satisfactory diagnostic accuracies have been validated for the diagnosis of significant coronary plaque burden [6].

In our study we have investigated the relationship between severity of coronary artery disease and LV diastolic dysfunction and to how extent could the presence of severe diastolic dysfunction predict the presence of obstructive coronary artery disease.

\section{Methods}

\subsection{Study Design}

This is a cross sectional observational study conducted during the period from June 2018 to June 2019 on 80 patients who underwent both coronary CTA and TTE within 1 year with no identifiable intervening cardiovascular event in $\mathrm{Na}-$ tional heart institute and Menoufia university hospitals.

\subsection{Study Population}

The exclusion criteria were LV ejection fraction $(\mathrm{LVEF})<45 \%$, moderate or se- 
vere valvular disease, other primary myocardial pathology (eg, hypertrophic cardiomyopathy, restrictive cardiomyopathy), significant pericardial disease (eg, constriction), congenital heart disease, primary pulmonary hypertension, and atrial or ventricular arrhythmias at the time of imaging, contraindications to coronary CT angiography testing.

All patients were subjected to informed consent and evaluated as regards to: Full clinical assessment ( Detailed medical history such as Age, gender, smoking, hypertension, diabetes, hypercholesterolemia), Physical Examination, 12 lead surface ECG, Laboratory investigation as creatinine, lipid Profile, $\mathrm{CBC}$ parameters, echocardiography examination including tissue Doppler imaging on mitral annulus and MSCT coronary angiography, the CT scan results were classified into non obstructive coronary artery disease (with maximum degree of stenosis in the coronaries $<50 \%$ in any major vessel ) and (obstructive CAD with maximal degree of stenosis $>50 \%$ )

According to the results our study included two groups:

Group I: included patients with non-obstructive coronary artery disease.

Group II: included patients with obstructive coronary artery disease.

\subsection{Statistical Analysis}

Data were analyzed using Statistical Program for Social Science (SPSS) version 25.0 for windows (SPSS Inc., Chicago, IL, USA) and NCSS 12.0 for windows (NCSS LCC., Kaysville, UT, USA).

Quantitative data of normal distribution were expressed as mean \pm standard deviation (SD). Median and inter-quartile range (IQR) were also calculated for quantitative data with abnormal distribution. Qualitative data were expressed as frequency and percentage.

The following tests were done:

Independent-samples t-test of significance was used when comparing between two means of normally distributed data.

Mann Whitney $U$ test is used to compare differences between two independent groups when the dependent variable is continuous, but not normally distributed.

Chi-square (X2) test also called Pearson's chi-square test or the chi-square test of association is used to discover if there is a relationship between two categorical variables.

Fisher Exact test is a test of significance that is used in the place of chi square test in 2 by 2 tables, especially in cases of small samples.

The "Linear-by-Linear" test is for ordinal (ordered) categories and assumes equal and ordered intervals. The Linear-by-Linear Association test is a test for trends in a larger than $2 \times 2$ table.

Multivariate regression analysis is used when we want to predict the value of a variable based on the value of two or more other variables. The variable we want to predict is called the dependent variable (or sometimes, the outcome, target or 
criterion variable). The variables we are using to predict the value of the dependent variable are called the independent variables (or sometimes, the predictor, explanatory or regressor variables). Results were represented in tables. The level of significance was considered statistically significant if $(\mathrm{P}$ value is $<0.05)$ and was high statistically signification if ( $\mathrm{P}$-value is $<0.01$ ), While $\mathrm{P}$ value $>0.05$ was considered non-significant.

\section{Results}

The 80 patients were classified into two groups:

Group one: patients with non-obstructive coronary artery disease $(n=38)$.

Group two: patients with obstructive coronary artery disease $(n=42)$.

\subsection{Regarding Risk Factors and Laboratory Data}

From 80 patients, 41 (51.2\%) of the patients were male, and mean age of patients was $55.9 \pm 8.0$ years, $35(43.8 \%)$ patients were hypertensive, $36(45 \%)$ patients were diabetic and $24(30 \%)$ patients were smokers. Regarding Age, patients in obstructive coronary artery disease group were significantly older compared to those in controls $(\mathrm{p}=0.028)$ [Table 1]. The proportion of male patients were higher in obstructive group 27 (64.3\%) than non-obstructive group 14 (36.8\%), $(\mathrm{P}=0.014)$ [Figure 1]. Diabetic cases also were higher in obstructive CAD patients 24 (57.1\%) than non-obstructive CAD 12 (31.6\%), also hypertension was more prevalent in obstructive CAD than non-obstructive $23(54.8 \%)$ and 12 (31.6\%) respectively. On the other hand, there was no significant relationship between study groups and smoking, dyslipidemia and family history $(\mathrm{P}=0.241$, $\mathrm{p}=0.181, \mathrm{p}=0.768$ respectively) [Table 1$]$.

\subsection{Regarding Assessment of Diastolic Dysfunction}

The relation between the degree of CAD either obstructive or non-obstructive assessed by MSCT coronary angiography and diastolic dysfunction detected by tissue Doppler assessment of mitral annular velocities. There are statistically significant relationship between septal e', lateral e', E/E', E/A, LAVI and TR velocity and the degree of $\mathrm{CAD}$, with higher values of those parameters in patients with obstructive CAD than patients with non-obstructive CAD ( $\mathrm{p} \leq 0.001,0.017$, $0.005,0.029,<0.001,0.034)$ respectively [Table 2].

In the other hand, the ejection fraction was significantly lower in patients with obstructive coronary artery disease group compared to non-obstructive CAD ( $\mathrm{p}$ $=0.027)$. DT (deceleration time) and IVRT (isovolumetric relaxation time) were also found to be significantly lower in the obstructive CAD group ( $\mathrm{p} \leq 0.001$, 0.004) respectively [Table 3].

The number of patients with diastolic dysfunction was significantly higher in the obstructive CAD group $(\mathrm{n}=35)$ in comparison to non-obstructive group ( $\mathrm{n}$ $=19)(\mathrm{p}$ value $=0.001)$ with higher number of patients with advanced grades of diastolic dysfunction (grade 2 and 3 ) in the obstructive group [Table 4]. 
Table 1. Comparison between the studied groups regarding the baseline characteristics.

\begin{tabular}{cccc}
\hline Baseline characteristics & Non-obstructive CAD & Obstructive CAD & $\begin{array}{c}\text { P-value } \\
\text { (Sig.) }\end{array}$ \\
\hline Count & 38 & 42 & \\
Mean \pm SD & Age (years) & & $0.028(\mathrm{~S})$ \\
& $53.9 \pm 7.8$ & $57.8 \pm 7.8$ & \\
Mean \pm SD & BMI (kg/m $\left.{ }^{2}\right)$ & & $0.728(\mathrm{NS})$ \\
& $26.9 \pm 3.3$ & $27.1 \pm 2.9$ & \\
Male gender & Risk factors & & $0.014(\mathrm{~S})$ \\
DM & $14(36.8 \%)$ & $27(64.3 \%)$ & $0.022(\mathrm{~S})$ \\
HTN & $12(31.6 \%)$ & $24(57.1 \%)$ & $0.037(\mathrm{~S})$ \\
Smoking & $12(31.6 \%)$ & $23(54.8 \%)$ & $0.241(\mathrm{NS})$ \\
Dyslipidemia & $9(23.7 \%)$ & $15(35.7 \%)$ & $0.181(\mathrm{NS})$ \\
Family history & $10(26.3 \%)$ & $17(40.5 \%)$ & $0.768(\mathrm{NS})$ \\
\hline
\end{tabular}

Table 2. Comparison between the studied groups regarding the mitral annular velocities using tissue Doppler echocardiography E/A ratio, TR velocity and LAVI.

\begin{tabular}{|c|c|c|c|}
\hline Echocardiographic data & Non-obstructive CAD & Obstructive CAD & P-value (Sig.) \\
\hline Count & 38 & 42 & \\
\hline \multicolumn{4}{|c|}{ Septal e' (m/s) } \\
\hline Mean \pm SD & $0.09 \pm 0.02$ & $0.07 \pm 0.02$ & $<0.001$ (HS) \\
\hline \multicolumn{4}{|c|}{ Lateral e' (m/s) } \\
\hline Mean \pm SD & $0.14 \pm 0.11$ & $0.10 \pm 0.02$ & $0.017(\mathrm{~S})$ \\
\hline \multicolumn{4}{|c|}{$E / e^{\prime}$} \\
\hline Mean \pm SD & $8.1 \pm 2.8$ & $10.6 \pm 4.6$ & $0.005(S)$ \\
\hline \multicolumn{4}{|c|}{$\mathrm{E} / \mathrm{A}$} \\
\hline Mean \pm SD & $1.26 \pm 0.55$ & $1.64 \pm 0.91$ & $0.029(\mathrm{~S})$ \\
\hline \multicolumn{4}{|c|}{ LAVI $\left(\mathrm{mL} / \mathrm{m}^{2}\right)$} \\
\hline Mean \pm SD & $30.6 \pm 4.5$ & $35.9 \pm 8.4$ & $<0.001$ (HS) \\
\hline \multicolumn{4}{|c|}{ TR velocity $(\mathrm{m} / \mathrm{s})$} \\
\hline Mean \pm SD & $1.7 \pm 0.9$ & $2.1 \pm 1.1$ & $0.034(\mathrm{~S})$ \\
\hline
\end{tabular}

Table 3. Comparison between the studied groups regarding the other echocardiographic data.

\begin{tabular}{cccc}
\hline Echocardiographic data & Non-obstructive CAD & Obstructive CAD & P-value (Sig.) \\
\hline Count & 38 & 42 & \\
Mean \pm SD & EF (\%) & & \\
& $61.8 \pm 8.3$ & & \\
Mean \pm SD & DT (ms) & $165 \pm 4.6 \pm 8.4$ & $<0.001(\mathrm{HS})$ \\
Mean \pm SD & $215 \pm 53$ & $100 \pm 24$ & $0.004(\mathrm{~S})$ \\
& IVRT (ms) & & \\
& $117 \pm 27$ & $18(42.9 \%)$ & $0.196(\mathrm{NS})$ \\
\hline
\end{tabular}


Table 4. Comparison between the 2 groups regarding diastolic dysfunction grading.

\begin{tabular}{cccc}
\hline Echocardiographic data & Non-obstructive CAD & Obstructive CAD & $\begin{array}{c}\text { P-value } \\
\text { (Sig.) }\end{array}$ \\
\cline { 1 - 2 } Count & 38 & 42 & \\
\hline No DD & Diastolic dysfunction & & \\
Grade 1 & $19(50 \%)$ & $7(16.7 \%)$ & \\
Grade 2 & $8(21.1 \%)$ & $10(23.8 \%)$ & \\
Grade 3 & $8(21.1 \%)$ & $13(31 \%)$ & \\
\hline
\end{tabular}

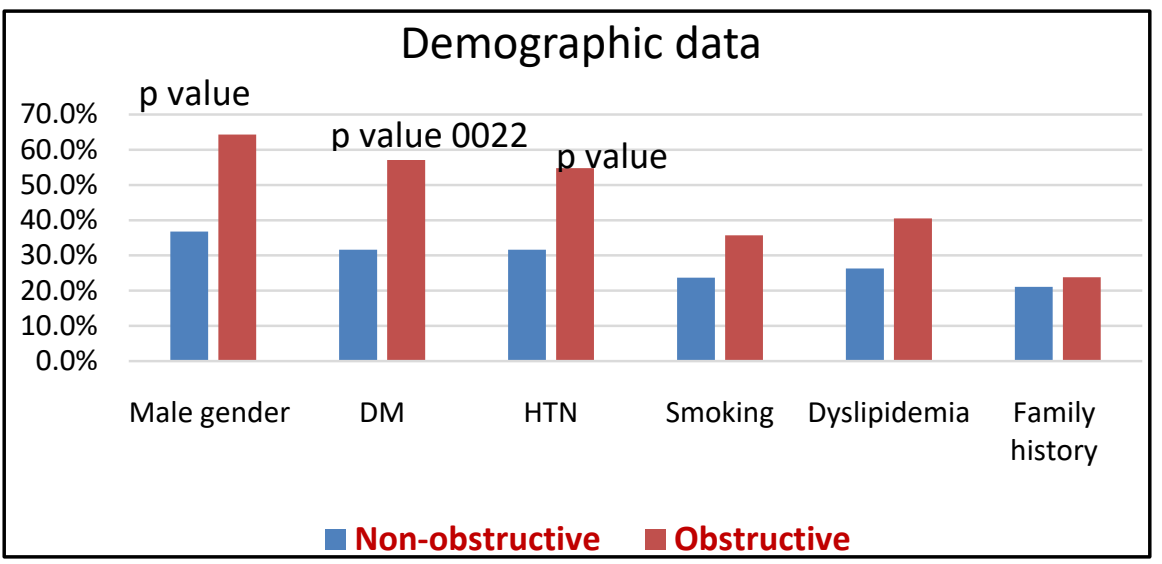

Figure 1. Comparison of the obstructive and non-obstructive CAD according to demographic data.

In the univariate analysis across the study, age, DM, HTN and male sex as risk factors were found to be statistically significant with degree of CAD Obstruction, as well as E/E', EF, LAVI and TR velocity by echocardiography, After multivariable adjustment that considered age, CAD risk factors an independent association was found between obstructive CAD and the presence of diastolic dysfunction with higher parameters E/E' and LAVI [Figure 2]. Tricuspid regurge velocity (TRV) and ejection fraction (EF) weren't found to have significant relationship with severity of CAD obstruction [Table 5, Table 6].

\section{Discussion}

In our study we have investigated the relationship between severity of coronary artery disease and LV diastolic dysfunction and to how extent could the presence of severe diastolic dysfunction predict the presence of obstructive coronary artery disease.

We divided the study into two groups based on the MSCT coronary angiography finding as 2 groups, first group is patients with non-obstructive coronary artery disease (maximum degree of stenosis in coronaries $<50 \%$ ), and second group is patients with obstructive coronary artery disease (maximum degree of stenosis $>50 \%$ ) and we compared both groups regarding risk factors of coronary artery disease and diastolic dysfunction, echocardiographic parameters to diagnosis 


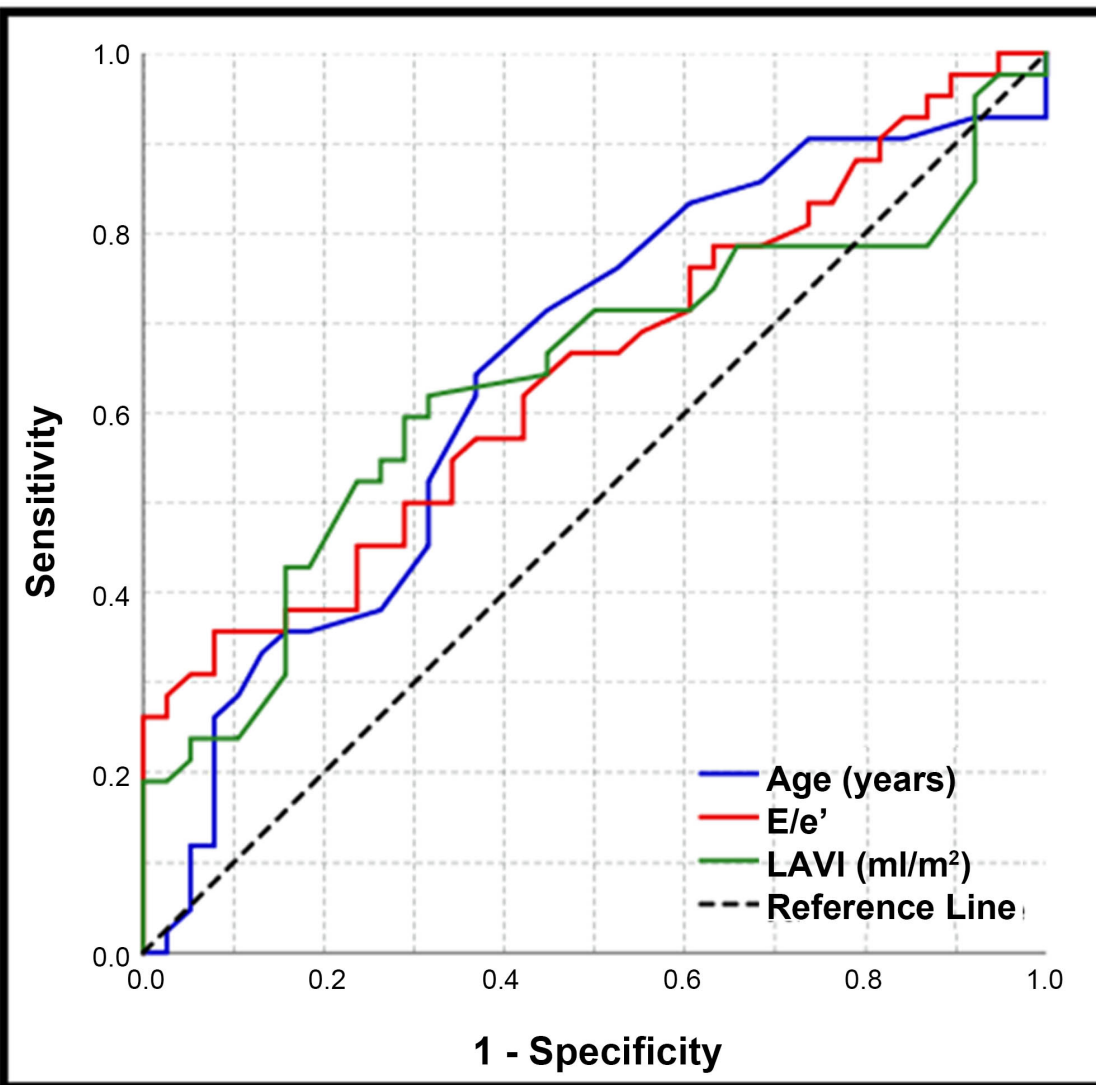

Figure 2. ROC curve analysis for the independent predictors of obstructive CAD.

Table 5. Univariate regression analysis for incidence of obstructive CAD.

\begin{tabular}{ccccc}
\hline \multirow{2}{*}{ Variable } & Unadjusted OR & \multicolumn{2}{c}{$95 \%$ Confidence Interval for OR } & P-value \\
\cline { 3 - 4 } & 1.067 & 1.006 & 1.132 & 0.032 \\
Age (years) & 3.086 & 1.239 & 7.686 & 0.016 \\
Male gender & 2.889 & 1.154 & 7.229 & 0.023 \\
DM & 2.623 & 1.051 & 6.548 & 0.039 \\
HTN & 0.939 & 0.887 & 0.995 & 0.033 \\
EF (\%) & 1.197 & 1.047 & 1.367 & 0.008 \\
E/e' & 1.091 & 1.015 & 1.172 & 0.018 \\
LAVI (mL/m & 1.595 & 1.049 & 2.425 & 0.029 \\
\hline TR velocity $(\mathrm{m} / \mathrm{s})$ & & & & \\
\hline
\end{tabular}

and classify diastolic dysfunction. Our study shows highly significance correlation between diastolic dysfunction degree and presence of obstructive coronary artery disease [Figure 3], according to diastolic dysfunction parameters, we found tissue Doppler imaging of mitral annular velocities data such as septal e', lateral e', and E/E' were significantly higher in the obstructive CAD group than non-obstructive CAD [Table 2] also IVRT, and deceleration time are significantly lower in the same group [Table 3]. 
Table 6. Multivariate regression analysis for incidence of obstructive CAD.

\begin{tabular}{ccccc}
\hline \multirow{2}{*}{ Variable } & Unadjusted OR & \multicolumn{2}{c}{ 95\% Confidence Interval for OR } & P-value \\
\cline { 3 - 4 } & 1.095 & 1.010 & 1.186 & 0.027 \\
Age (years) & 6.340 & 1.505 & 26.699 & 0.012 \\
Male gender & 10.401 & 2.027 & 53.356 & 0.005 \\
DM & 5.062 & 1.118 & 22.919 & 0.035 \\
HTN & 0.956 & 0.880 & 1.038 & 0.286 \\
EF (\%) & 1.395 & 1.078 & 1.805 & 0.011 \\
E/e' & 1.132 & 1.003 & 1.277 & 0.044 \\
LAVI (mL/m & & & Upper Bound & \\
TR velocity $(\mathrm{m} / \mathrm{s})$ & 1.067 & 0.426 & 2.675 & 0.890 \\
\hline
\end{tabular}

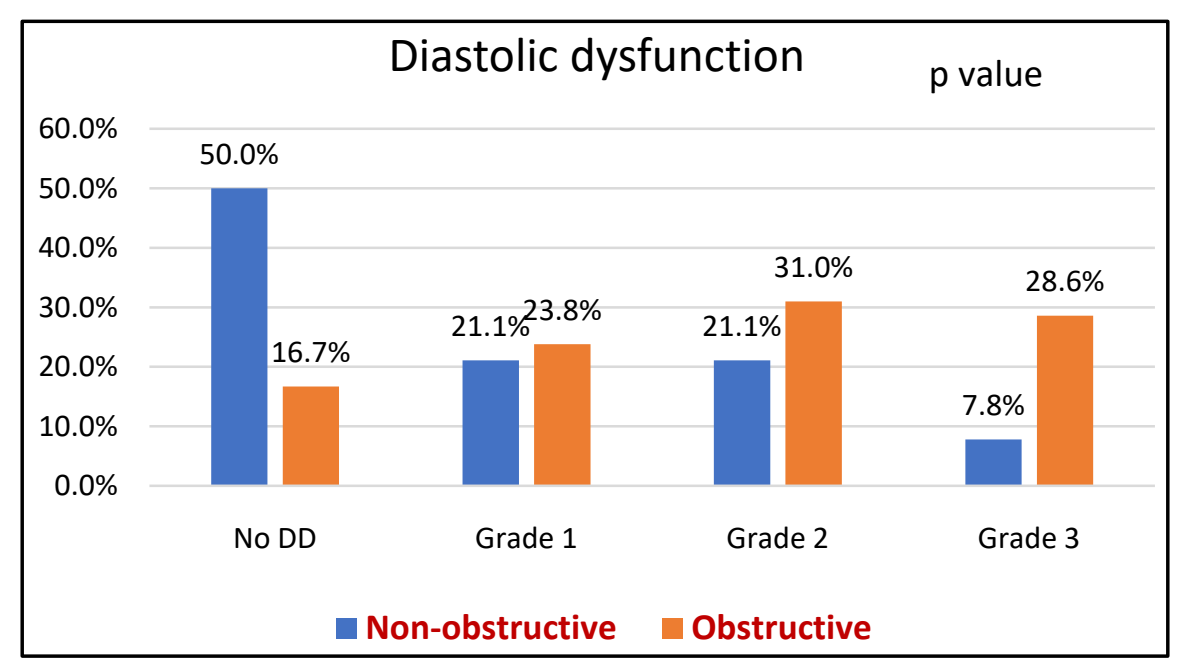

Figure 3. Comparison between obstructive and non-obstructive CAD regarding diastolic dysfunction degree.

Left atrial volume index (LAVI) and tricuspid regurge velocity (TRV) are two important factors in the diagnosis of diastolic dysfunction according to latest guidelines. The present study compared both groups regarding those factors and LAVI and TRV are found to be high in the obstructive group with statistical significance [Table 2].

Estimation of cardiac systolic function of LV has also been compared between the groups and we found ejection fraction to be significantly lower in the obstructive group. According to risk factors of coronary artery disease, we found that hypertension, diabetes, and male sex are more common in patients with obstructive coronary artery disease with statistical significance.

These result are in agreement with the results of many studies like fay $\mathrm{Y}$ et al., the study observed a direct relationship between DD degree and LVEDP and the extent and severity of noninvasively assessed CAD burden by the presence of obstructive coronary artery disease as well as number of coronary vessels with obstructive CAD. There were significantly higher values of septal e', lateral e' 
and $\mathrm{E} / \mathrm{E}$ ' in patients with higher burden of coronary artery disease. Lower values of IVRT and Deceleration time were also found among patients were more severe obstructive CAD [7].

Abhayaratna et al. is a study to detect significance of CAD and presence of preclinical diastolic they found increase incidence of diastolic dysfunction among patients with coronary artery disease and myocardial infarction. There were highly significant correlation between $\mathrm{CAD}$ and preclinical diastolic dysfunction [8].

In agreement with our study the result of Ren et al. who found that CAD is considered an independent risk factor for developing diastolic dysfunction [9].

Vlasseros, I. et al. investigated the combined effects of hypertension and coronary artery disease (CAD) on left ventricular (LV) diastolic function. The study participants were divided into two groups according to the presence of CAD by coronary angiography. Patients with CAD exhibited lower ejection fraction. The main finding of this study was that severely depressed LV diastolic function, as suggested by various echocardiographic indices, is indicative of CAD coexistence in patients with uncomplicated essential hypertension., In hypertensive, the early recognition of LV diastolic performance alteration may be associated with the presence of significant CAD [10].

Prior studies assessing the association between DD and CAD came to conflicting conclusions. Garcia et al. demonstrated that early stage of subclinical atherosclerotic disease is negatively associated with DD parameters [11]. Only 48 subjects included in the study, no tissue Doppler assessment of mitral annular velocity was done, and carotid intima-media thickness was used as a surrogate for subclinical atherosclerosis instead of presence of coronary artery disease by MSCT.

Eleid et al. did not find consistent relation between coronary artery plaque burden as assessed by coronary calcium score and echocardiographic grade of LV DD [12]. Their study population had lower risk factor profile than our study: Only $6 \%$ are diabetic and $36 \%$ hypertensive furthermore the study only assessed coronary artery disease by calcium score without angiographic evidence of luminal narrowing.

In the other hand Abdelrahman J., et al. is another cross sectional study. the study suggests that CACS, as well as CAD by CCTA, are not independently associated with measures of DD on echocardiography [13].

The difference of the result could be explained by more than half of the former study subjects have prevalent $\mathrm{CAD}$ in the form of angina, previous myocardial infarction, or previous coronary artery bypass surgery, on the contrary our study excluded subjects with previous MI or CABG.

\section{Study Limitations}

Although the number of patients enrolled in our study is relatively small, the results are quite comparable to larger studies. 


\section{Conclusion}

Our study showed that obstructive coronary artery disease has major implications on diastolic function of the heart and the extent and severity of CAD by coronary CTA which is associated with worsening diastolic function.

\section{Funding}

The author(s) received no financial support for the research, authorship, and/or publication of this article.

\section{Conflicts of Interest}

The authors declared no potential conflicts of interest with respect to the research, authorship, and/or publication of this article.

\section{References}

[1] Owan, T.E. and Redfield, M.M. (2005) Epidemiology of Diastolic Heart Failure. Progress in Cardiovascular Diseases, 47, 320-332. https://doi.org/10.1016/j.pcad.2005.02.010

[2] Ahmed, A., Perry, G.J., Fleg, J.L., Love, T.E., Goff Jr., D.C. and Kitzman, D.W. (2006) Outcomes in Ambulatory Chronic Systolic and Diastolic Heart Failure: A Propensity Score Analysis. American Heart Journal, 152, 956-966. https://doi.org/10.1016/j.ahj.2006.06.020

[3] Aroesty, J.M., McKay, R.G., Heller, G.V., Royal, H.D., Als, A.V. and Grossman, W.I.L.L.I.A.M. (1985) Simultaneous Assessment of Left Ventricular Systolic and Diastolic Dysfunction during Pacing-Induced Ischemia. Circulation, 71, 889-900. https://doi.org/10.1161/01.CIR.71.5.889

[4] Oh, J.K., Park, S.J. and Nagueh, S.F. (2011) Established and Novel Clinical Applications of Diastolic Function Assessment by Echocardiography. Circulation: Cardiovascular Imaging, 4, 444-455. https://doi.org/10.1161/CIRCIMAGING.110.961623

[5] Ndrepepa, G. (2017) Atherosclerosis \& Ischaemic Heart Disease: Here to Stay or Gone Tomorrow. The Indian Journal of Medical Research, 146, 293-297.

[6] Kishi, S., Magalhaes, T.A., Cerci, R.J., Matheson, M.B., Vavere, A., Tanami, Y., Kitslaar, P.H., George, R.T., Brinker, J., Miller, J.M. and Clouse, M.E. (2016) Total Coronary Atherosclerotic Plaque Burden Assessment by CT Angiography for Detecting Obstructive Coronary Artery Disease Associated with Myocardial Perfusion Abnormalities. Journal of Cardiovascular Computed Tomography, 10, 121-127. https://doi.org/10.1016/j.jcct.2016.01.005

[7] Lin, F.Y., Zemedkun, M., Dunning, A., Gomez, M., Labounty, T.M., Asim, M., et al. (2013) Extent and Severity of Coronary Artery Disease by Coronary CT Angiography Is Associated with Elevated Left Ventricular Diastolic Pressures and Worsening Diastolic Function. Journal of Cardiovascular Computed Tomography, 7, 289-296. https://doi.org/10.1016/j.jcct.2013.08.008

[8] Abhayaratna, W.P., Marwick, T.H., Smith, W.T. and Becker, N.G. (2006) Characteristics of Left Ventricular Diastolic Dysfunction in the Community: An Echocardiographic Survey. Heart (British Cardiac Society), 92, 1259-1264. https://doi.org/10.1136/hrt.2005.080150

[9] Ren, X., Ristow, B., Na, B., Ali, S., Schiller, N.B. and Whooley, M.A. (2007) Preva- 
lence and Prognosis of Asymptomatic Left Ventricular Diastolic Dysfunction in Ambulatory Patients with Coronary Heart Disease. The American Journal of Cardiology, 99, 1643-1647. https://doi.org/10.1016/j.amjcard.2007.01.041

[10] Vlasseros, I., Katsi, V., Vyssoulis, G., Pylarinos, I., Richter, D., Gialernios, T., et al. (2013) Aggravation of Left Ventricular Diastolic Dysfunction in Hypertensives with Coronary Artery Disease. Hypertension Research, 36, 885-888.

https://doi.org/10.1038/hr.2013.67

[11] Garcia, M.M.O., Rodrigues, M.G., Reis Neto, J.A.D. and Correia, L.C. (2010) Influence of Subclinical Atherosclerosis on Diastolic Function in Individuals Free of Cardiovascular Fisease. Arquivos Brasileiros de Cardiologia, 95, 473-479. https://doi.org/10.1590/S0066-782X2010005000114

[12] Eleid, M.F., Appleton, C.P., Lopez, A.G., Cha, S. and Hurst, R.T. (2011) Coronary Artery Plaque Burden Does not Affect Left Ventricular Diastolic Function in Asymptomatic Adults with Normal Ejection Fraction. Journal of the American Society of Echocardiography, 24, 909-914. https://doi.org/10.1016/j.echo.2011.03.017

[13] Jamiel, A., Ahmed, A.M., Farah, I. and Al-Mallah, M.H. (2016) Correlation between Diastolic Dysfunction and Coronary Artery Disease on Coronary Computed Tomography Angiography. Heart Views: The Official Journal of the Gulf Heart Association, 17, 13-18. https://doi.org/10.4103/1995-705X.182649 